\title{
ANÁLISE COMPARATIVA DO EQUILÍBRIO QUASE ESTÁTICO ENTRE PRATICANTES DE MUAY-THAI DE DIFERENTES NÍVEIS DE APRENDIZADO
}

\section{Patricia Aparecida Brigida}

Graduada em Educação Física pela Faculdade Metropolitana de Maringá (FAMMA), Maringá (PR), Brasil.

\section{Fernando Possamai}

Graduado em Educação Física pela Universidade Estadual de Maringá (UEM), Maringá (PR), Brasil.

José Roberto Andrade do Nascimento Júnior

Doutor em Educação Física pela Universidade Estadual de Maringá (UEM); Docente adjunto no departamento de Educação Física da Universidade Federal do Vale do São Francisco (UNIVASF), Brasil.

\section{Weslei Jacob}

Doutorando em Atividade Física, Educação Física e Esporte, linha de pesquisa: Treinamento Esportivo, pela Universidade de Barcelona-Espanha.

\section{Daniel Vicentini de Oliveira}

Doutorando em Gerontologia pela Faculdade de Ciências Médicas da Universidade Estadual de Campinas (UNICAMP); Docente adjunto no departamento de Educação física da Faculdade Metropolitana de Maringá (FAMMA), Maringá (PR) Brasil.
RESUMO: Este estudo teve como objetivo analisar e comparar o equilíbrio quase estático de praticantes de Muay-Thai de diferentes níveis de aprendizado. Trata-se de um estudo transversal, no qual a amostra foi constituída de 20 praticantes de Muay-Thai, sendo 10 do Grupo Iniciante e 10 do Grupo Avançado de aprendizagem da modalidade. $O$ instrumento utilizado para a coleta de dados foi a plataforma de força da marca EMG System do Brasil, modelo Biomec 4000. O protocolo aplicado foi a Estabilometria. Cada indivíduo realizou os testes nas condições: bipodal com os olhos fechados (BOF) e olhos abertos (BOA), e na posição monopodal com olhos fechados (MOF) e olhos abertos (MOA). Os dados foram analisados por meio da estatística descritiva e inferencial. Por meio dos resultados, pode-se identificar diferença significativa $(p=0,04)$ na área MOA, evidenciando que os praticantes em nível avançado apresentam maior equilíbrio quase estático quando comparados com praticantes iniciantes. Concluise que é maior o equilíbrio quase estático na posição monopodal com olhos abertos dos praticantes de Muay-Thai em nível avançado, em comparação aos de nível inicial.

PALAVRAS-CHAVE: Aptidão física; Artes marciais; Exercício físico.

\section{COMPARATIVE ANALYSIS OF QUASI-STATIC EQUILIBRIUM AMONG MUAY-THAI FIGHTERS AT DIFFERENT LEARNING LEVELS}

ABSTRACT: The almost static equilibrium of Muay-Thai fighters at different learning levels is analyzed and compared. Current transversal study comprised 20 Muay-Thai fighters, with ten from the Initiating Group and ten from the Advanced Group of learners. Data were collected by force platform EMG System do Brasil, model Biomec 4000 , with the application of Stable-meter protocol. Each agent underwent tests within the bipodal condition with closed (BOF) and open (BOA) eyes and within the monopodal position with closed (MOF) and open (MOA) eyes. Descriptive and inferential statistics evaluated the data. Results identified significant differences $(p=0.04)$ in MOA and revealed that fighters at the advanced level had a greater quasi-equilibrium when compared to initiating ones. Quasi-static equilibrium is greater in the monopodal position with open eyes of Muay-Thai fighters at an advanced level when compared to those at the initial level.

KEY WORDS: Physical capacity; Martial arts; Physical exercise. 


\section{INTRODUÇão}

Diante de diversas modalidades esportivas, as lutas vêm apresentando grande crescimento em diversos países. Dentre elas, destaca-se o Muay-Thai, uma arte marcial de origem tailandesa com mais de 2.000 anos que ainda é praticada no país e vem conquistando adeptos pelo mundo, tornando-se cada vez mais popularizada e conhecida pelo fato de ser uma das lutas mais utilizadas atualmente no Mix Martial Arts (M. M. A.) (ROCHA et al., 2013).

O Muay-Thai é considerado uma modalidade complexa, pois se constitui de combinações de golpes caracterizados pelo uso de punhos, joelhos, cotovelos, chutes (lateral e frontal) e arremessos (DUARTE, 2004). A função em utilizar vários segmentos corporais para execução de golpes de ataque e defesa tornam alguns componentes técnicos indispensáveis para um bom desempenho, conforme cita Falkenbach (2007), afinal os atletas necessitam de muita resistência, força, agilidade, coordenação e o equilíbrio.

O equilíbrio, segundo Bankoff et al. (2006), está relacionado entre as forças que agem sobre o corpo durante ações motoras buscando, assim, uma postura corporal adequada, ou seja, a cada movimento adotado pelos indivíduos, novas informações e respostas são recebidas e necessárias para que se mantenha o equilíbrio do corpo. Sabe-se que essas respostas são formadas a partir da informação sensorial de três sistemas, o visual, o vestibular e o somatossensorial (MOCHIZUKI; AMADIO, 2006).

Os sistemas responsáveis pelo equilíbrio citados acima atuam de forma integrada e diferenciada para cada perturbação que age sobre o corpo, no qual o visual fornece informações sobre a posição e movimento de um objeto no espaço, o somatossensorial, ou proprioceptivo como também é conhecido, vai fornecer informações sobre a posição corporal em relação à superfície de suporte, e o vestibular é responsável por transmitir informações sobre a posição e movimento da cabeça em relação às forças que atuam sobre o corpo (DUARTE, 2000).

A manutenção do equilíbrio é essencial para a execução de atividades físicas da vida diária e também de grande importância para a prática de atividades esportivas. Em algumas modalidades, o nível de equilíbrio do atleta pode interferir diretamente nos resultados, tornando o seu estudo necessário para prescrição de treinamentos adequados, uma vez que essa qualidade física pode, direta ou indiretamente, afetar o desempenho esportivo.

A análise do equilíbrio quase estático poderá resultar em comparações dessa natureza entre indivíduos iniciantes e avançados da modalidade esportiva MuayThai e o quanto esses praticantes deslocam o Centro de Pressão (CP) quando em posição estática. É válido citar que segundo Duarte e Freitas (2010), mesmo parado o corpo sofre oscilações, isso porque forças externas (força gravitacional e a força de reação do solo) e as forças internas (perturbações fisiológicas por exemplo), atuam sobre o corpo humano, onde do ponto de vista mecânico, o corpo nunca está em perfeito estado de equilíbrio.

O CP corresponde ao ponto de aplicação das forças verticais agindo sobre a superfície de suporte. De forma simplificada, a tarefa do sistema de controle postural é manter a projeção horizontal do Centro de Gravidade (CG) do indivíduo dentro da base de suporte definida pela área da base dos pés (DUARTE, 2000).

$\mathrm{O}$ equipamento mais utilizado para mensurar o equilíbrio de um corpo por meio do $\mathrm{CP}$, sendo essa a variável mais comum, é a plataforma de força, que consiste em uma placa sob a qual alguns sensores estão posicionados para medir componentes de força em três direções (anteroposterior, médio-lateral e vertical). Essa avaliação da oscilação corporal é realizada por meio de um estudo, a Estabilometria, criado para medir a habilidade de manter a postura ereta (DUARTE, 2000; DUARTE; FREITAS, 2010).

Estudos relacionados com equilíbrio são bastante frequentes no sentido de compreender os sistemas responsáveis por tal habilidade corporal. Compreender as variáveis que podem ser treinadas e avaliadas para melhorar e manter o equilíbrio corporal se faz importante e necessário para predizer treinamento e estabelecer comparações entre indivíduos treinados e não treinados.

Posto isso, o presente estudo visa responder à seguinte questão norteadora: existe diferença no equilíbrio quase estático em praticantes de Muay-Thai de diferentes níveis de aprendizado? Este estudo teve como 
objetivo analisar e comparar o equilíbrio quase estático de praticantes de Muay-Thai de diferentes níveis de aprendizado.

\section{MÉTODOS}

O presente estudo tem caráter quantitativo, observacional e transversal, aprovado pelo Comitê de Ética em Pesquisa com Seres Humanos do Centro Universitário Cesumar (UniCesumar) por meio do parecer $\mathrm{n}^{\circ} 1.432 .642$.

A população investigada consistiu-se em praticantes da modalidade de Muay-Thai, do sexo masculino, de uma academia da cidade de Maringá, Estado do Paraná. A amostra não probabilística foi escolhida de forma intencional, na qual foi composta por 20 praticantes da modalidade, com média de idade de 24,60 66,63 anos, peso médio $80,92 \pm 14,08$ quilogramas e estatura média de 175,45 $\pm 7,96$ centímetros, divididos igualmente em dois grupos de 10 participantes, que correspondiam aos níveis de aprendizado iniciante e avançado (Grupo Iniciante e Grupo Avançado). Como critério de inclusão, os participantes não poderiam apresentar histórico de lesão em membros inferiores, o que foi questionado por meio de uma anamnese. A exclusão de participantes do sexo feminino se explica pela baixa prevalência das mesmas nas aulas da modalidade na academia estudada.

Os participantes foram orientados sobre o estudo e a participação foi solicitada de forma democrática e livre. Todos receberam e assinaram o Termo de Consentimento Livre e Esclarecido (TCLE) de participação na pesquisa de cunho científico. Antes da aplicação dos testes foram coletadas as seguintes informações: idade, massa corpórea por meio de balança digital e balança antropométrica e estatura por meio do estadiômetro. O nível de aprendizado de cada indivíduo avaliado foi indicado pelos treinadores responsáveis pelo local de pesquisa.

O instrumento utilizado para a coleta de dados foi a plataforma de força da marca EMG System do Brasil, modelo Biomec 4000, cedida pelo Centro Integrado de Análise do Movimento (CIAM) da cidade de Maringá (PR). O protocolo aplicado foi a Estabilometria, que consiste em mapear e medir a trajetória do centro de pressão, estando o avaliado em posição quase estática sobre a base do instrumento plataforma de força.

Aplataformadeforçafoidevidamente posicionada em um ambiente adequado, livre de interferências visuais e sonoras, à sua frente foi posicionado um ponto localizando-se na mesma altura dos olhos do avaliado, o qual serviu de referência visual e postural durante o teste de equilíbrio sobre o instrumento biomecânico (técnica da Estabilometria). Os indivíduos avaliados não poderiam ter realizado atividades físicas intensas anteriormente aos testes.

Antes de iniciar as coletas com a plataforma de força, os participantes passaram por um período de familiarização, posicionando-se sobre o equipamento de medida nos moldes da atividade principal. Cada indivíduo realizou os testes em quatro situações distintas nas condições: bipodal com os olhos fechados (BOF) e olhos abertos (BOA), e na posição monopodal com olhos fechados (MOF) e olhos abertos (MOA), ambos com o membro inferior dominante do sujeito.

Para cada variação o indivíduo permaneceu sobre a plataforma de força, descalço, durante 30 segundos, respeitando um intervalo entre as variações de 30 a 40 segundos. Para os testes com olhos fechados foi solicitado que cada participante tentasse permanecer durante $o$ tempo de coleta com os olhos fechados (MELLO et al., 2002; IMBIRIBA et al., 2001).

Os dados coletados foram fornecidos por meio de um software específico da plataforma de força. Por meio de um software matemático uma rotina foi elaborada para retirar os dados desse instrumento, após a sua retirada foram tabulados em planilha eletrônica, sendo preparados para a sua análise.

A análise desses dados foi realizada por meio de estatística descritiva e inferencial, passando primeiramente por teste de normalidade utilizando o teste Shapiro-Wilk. Identificada a necessidade de utilizar a estatística não paramétrica, os dados foram apresentados em mediana (Md) e intervalos interquartílicos (Q1-Q3). Para a comparação entre os grupos iniciante e avançado, aplicou-se o teste "U" de Mann-Whitney, adotando valor de significância de $95 \%$. 


\section{RESULTADOS}

A Tabela 1 apresenta os resultados obtidos sobre a área e a amplitude do deslocamento anteroposterior e médio-lateral dos praticantes avaliados.

Tabela 1. Área e deslocamentos (médio-lateral e anteroposterior) do centro de pressão de praticantes de MuayThai

\begin{tabular}{ccc}
\hline POSICIONAMENTO & Md & Q1-Q3 \\
\hline BOA & 0,45 & $0,32-0,71$ \\
BOF & 0,53 & $0,33-0,71$ \\
MOA & 2,42 & $1,65-3,64$ \\
MOF & 11,88 & $6,38-16,36$ \\
ML_BOA & 0,85 & $0,61-1,04$ \\
ML_BOF & 0,98 & $0,80-1,04$ \\
ML_MOA & 2,01 & $1,77-2,72$ \\
ML_MOF & 4,89 & $3,43-7,63$ \\
AP_BOA & 1,19 & $1,06-1,73$ \\
AP_BOF & 1,36 & $1,16-1,89$ \\
AP_MOA & 2,61 & $1,93-2,87$ \\
AP_MOF & 5,36 & $3,71-6,63$ \\
\hline
\end{tabular}

Legenda: BOA: bipodal olhos abertos; BOF: bipodal olhos fechados; MOA: monopodal olhos abertos; MOF: monopodal olhos fechados; ML_BOA: médio-lateral bipodal olhos abertos; ML_BOF: médio-lateral bipodal olhos fechados; ML_MOA: médio-lateral monopodal olhos abertos; ML_MOF: médio-lateral monopodal olhos fechados; AP_BOA: anteroposterior bipodal olhos abertos; AP_BOF: anteroposterior bipodal olhos fechados; AP_MOA: anteroposterior monopodal olhos abertos; AP_MOF: anteroposterior monopodal olhos fechados.

Os resultados acima indicam que a oscilação corporal na posição BOF é maior quando comparado a BOA. Essa oscilação se torna ainda mais evidente quando a posição do indivíduo é monopodal. Quando voltado para o deslocamento, os valores obtidos na direção anteroposterior também se mostram superiores.

Por meio dos resultados apresentados na Tabela 2 , pode-se identificar diferença significativa $(p=0,04)$ na área MOA, evidenciando que os praticantes em nível avançado apresentam maior equilíbrio quase estático quando comparados com praticantes iniciantes.
Tabela 2. Comparação da área monopodal (olhos abertos e fechados) e bipodal (olhos abertos e fechados) entre praticantes iniciantes e avançados de Muay-Thai

\begin{tabular}{cccc}
\hline \multirow{2}{*}{$\begin{array}{c}\text { Área } \\
\left(\mathbf{c m}^{2}\right)\end{array}$} & \multicolumn{2}{c}{ Md (Q1-Q3) } & $\boldsymbol{P}$ \\
\cline { 2 - 3 } & Iniciante & Avançado & \\
\hline BOA & $0,48(0,33-0,74)$ & $0,45(0,29-0,73)$ & 0,65 \\
BOF & $0,54(0,35-0,73)$ & $0,49(0,29-0,79)$ & 0,94 \\
MOA & $3,03(2,28-3,95)$ & $1,69(1,51-2,61)$ & $\mathbf{0 , 0 4 *}$ \\
MOF & $11,88(7,35-15,56)$ & $11,72(4,86-27,75)$ & 0,94 \\
\hline
\end{tabular}

*diferença significativa $p<0,05$.

Legenda: BOA: bipodal olhos abertos; BOF: bipodal olhos fechados; MOA: monopodal olhos abertos; MOF: monopodal olhos fechados.

$\mathrm{Na}$ sequência, as tabelas de comparação do equilíbrio quase estático entre os dois níveis de aprendizado dos praticantes apresentam os resultados obtidos por meio da análise da amplitude dos deslocamentos médio-lateral e anteroposterior do centro de pressão nas quatro situações sugeridas.

Tabela 3. Comparação da amplitude do deslocamento médiolateral bipodal (olhos abertos e fechados) e monopodal (olhos abertos e fechados) entre praticantes iniciantes e avançados de Muay-Thai

\begin{tabular}{cccc}
\hline \multirow{2}{*}{$\begin{array}{c}\text { Deslocamento } \\
\text { médio-lateral } \\
(\mathbf{c m})\end{array}$} & \multicolumn{2}{c}{ Md (Q1-Q3) } & \multirow{2}{*}{ Iniciante } \\
\cline { 2 - 3 } & Avançado & \\
\hline ML_BOA & $0,86(0,79-1,06)$ & $0,84(0,69-1,07)$ & 0,70 \\
ML_BOF & $0,97(0,79-1,13)$ & $0,98(0,78-1,03)$ & 0,82 \\
ML_MOA & $1,89(1,65-2,07)$ & $2,34(1,89-2,87)$ & 0,09 \\
ML_MOF & $5,06(3,35-7,73)$ & $4,60(3,27-7,83)$ & 0,94 \\
\hline
\end{tabular}

*diferença significativa $p<0,05$.

Legenda: ML_BOA: médio-lateral bipodal olhos abertos; ML_BOF: médio-lateral bipodal olhos fechados; ML_MOA: médio-lateral monopodal olhos abertos; ML_MOF: médio-lateral monopodal olhos fechados.

De acordo com a Tabela 3, verifica-se que não há diferença na amplitude de deslocamento médio-lateral monopodal e bipodal, ambos com os olhos abertos $\mathrm{e}$ fechados, entre praticantes iniciantes e avançados de Muay-Thai. Na Tabela 4 observa-se que também não houve diferença na amplitude de deslocamento anteroposterior monopodal e bipodal. 
Tabela 4. Comparação da amplitude do deslocamento anteroposterior monopodal (olhos abertos e fechados) e bipodal (olhos abertos e fechados) entre praticantes iniciantes e avançados de Muay-Thai

\begin{tabular}{cccc}
\hline \multirow{2}{*}{$\begin{array}{c}\text { Deslocamento } \\
\text { anteroposterior } \\
(\mathbf{c m})\end{array}$} & \multicolumn{2}{c}{ Md (Q1-Q3) } & \multirow{2}{*}{$\boldsymbol{P}$} \\
\cline { 2 - 3 } & Iniciante & Avançado & \\
\hline AP_BOA & $1,23(1,02-1,79)$ & $1,19(1,10-1,84)$ & 0,70 \\
AP_BOF & $1,35(1,12-1,59)$ & $1,36(1,15-2,29)$ & 0,65 \\
AP_MOA & $2,16(1,76-2,86)$ & $2,84(1,97-2,88)$ & 0,23 \\
AP_MOF & $5,01(3,47-6,71)$ & $5,36(3,39-7,23)$ & 0,88 \\
\hline
\end{tabular}

*diferença significativa $p<0,05$.

Legenda: AP_BOA: anteroposterior bipodal olhos abertos; AP_BOF: anteroposterior bipodal olhos fechados; AP_MOA: anteroposterior monopodal olhos abertos; AP_MOF: anteroposterior monopodal olhos fechados.

Diante dos resultados levantados e apontados nas Tabelas 3 e 4, observou-se que não foram apresentadas diferenças significativas entre os grupos iniciante e avançado, demonstrando assim que o equilíbrio quase estático nas condições determinadas por meio do deslocamento médio-lateral e anteroposterior são semelhantes para ambos os grupos.

\section{DISCUSSÃO}

O equilíbrio é requisitado em diversas situações do dia a dia, e, em algumas delas, o indivíduo pode necessitar de mais ou menos estabilidade. Quando voltado aos esportes, pode-se observar variações no equilíbrio, como por exemplo na saída do bloco em uma corrida, no arremesso do basquetebol e nas lutas, como o judô (FERREIRA, 2003).

Quando tratados os resultados que avaliaram a área e a amplitude do deslocamento médio-lateral e anteroposterior, notou-se que os praticantes tiveram um maior desequilíbrio quando testados em situação monopodal com a suspensão do sistema visual. Um estudo com jovens participantes de atividades físicas regulares para verificação das implicações da informação visual sobre a amplitude do deslocamento nas direções anteroposterior e médio-lateral durante o equilíbrio estático, realizado por Teixeira et al. (2007), demonstrou que, mesmo em indivíduos praticantes de atividades físicas regulares, a informação visual é um sistema determinante para a manutenção do equilíbrio.

Esses resultados estão de acordo com a literatura, uma vez que diversos estudos apontam para um aumento da oscilação corporal quando é retirada alguma fonte de informação sensorial. Como exemplo, no estudo feito por Rougier (2003), o qual avaliou a influência da informação visual a partir do CP na plataforma de força, verificando que essa informação pode afetar tanto a oscilação do $\mathrm{CP}$, como a forma que o mesmo se comporta durante a correção da oscilação corporal. Buchanan e Horak (2003), também mencionados pelos mesmos autores, mostraram que o sistema visual contribui indiscutivelmente para manter o CP no centro da base de apoio.

Mesmo que o sistema de controle postural já esteja desenvolvido quando a informação visual é retirada, há aumento na área e no deslocamento do centro de força. Quando os indivíduos se encontram na condição de olhos fechados, a tarefa de manutenção do equilíbrio se torna mais complexa por ser uma situação pouco comum, assim, para a realização de adaptações necessárias, o controle postural é mais exigido levando a uma queda de performance (ALVES; BARELA, 2005).

Ainda contribuindo com esta tese, Kleiner, Schlittler e Sánchez- Arias (2011) citam que, durante a manutenção da postura ereta estática, a oscilação corporal aumenta até mais que o dobro quando a visão não está disponível, concluindo assim que esse sistema sensorial propicia uma melhora no desempenho do sistema de controle postural.

A amplitude de deslocamento na direção anteroposterior também se mostrou superior em relação à médio-lateral, onde também foi notória maior oscilação quando a informação visual não pode ser utilizada. Alguns estudos afirmam que na direção anteroposterior há a demanda de um maior controle, pois a manutenção do equilíbrio nesta direção depende de um número maior de graus de liberdade nas articulações quando comparado à direção médio-lateral (MANN et al., 2005).

Os resultados obtidos por meio da comparação da área nas condições de olhos abertos e fechados, em posição bipodal e monopodal, apontaram que apenas 
na avaliação da área na condição monopodal com olhos abertos houve diferença significativa entre os praticantes iniciantes e avançados no Muay-Thai, indicando que os praticantes avançados possuem maior equilíbrio quase estático.

Esse resultado do presente estudo vai ao encontro com o estudo de Paillard e Noe (2006), feito com jogadores de futebol para verificar a contribuição da informação visual em atletas profissionais e não profissionais. Os autores detectaram que, além dos atletas de alto nível apresentarem maior estabilidade, a contribuição visual para a manutenção do equilíbrio é menos importante para atletas profissionais. Defendido por Yoshitomi et al. (2006), em seu estudo com judocas mais e menos graduados e indivíduos sedentários, o nível de experiência favorece uma melhor condição de equilíbrio corporal.

Quando comparados os resultados acerca da amplitude de deslocamento médio-lateral e anteroposterior nas condições propostas, não houve nenhum resultado significativo entre os dois grupos. Esse resultado pode induzir ao raciocínio de que independente do tempo de prática no Muay-Thai, os praticantes adquirem maior equilíbrio, uma vez que essa capacidade física é comumente requisitada durante a execução da modalidade. Corroborando ainda com essa dedução, Vieira e Oliveira (2006) apontam que indivíduos bem condicionados apresentam menos deslocamento do CP e consequentemente um bom equilíbrio.

Neste âmbito, Barbati (1979) defende que o treinamento físico no Muay-Thai é de extrema necessidade, visto que a preparação física de forma geral e específica influencia na força, velocidade, flexibilidade, resistência, coordenação e equilíbrio do atleta. E ainda, na ausência de condição física ideal, o atleta tem dificuldades em realizar os movimentos que podem intervir de forma direta no resultado das lutas.

Os atletas e praticantes de esportes, e principalmente lutas, são submetidos a uma exigência motora fina, o que os tornam altamente dependentes dos sistemas sensório-motores responsáveis pelo controle do equilíbrio. Como consequência, atletas parecem apresentar melhor controle do equilíbrio do que não atletas. Do mesmo modo, atletas praticantes de modalidades que demandam maior exigência de membros inferiores tendem a apresentar padrões superiores de estabilidade (LOPES, 2013).
Parecem escassos os estudos que envolvem a prática do Muay-Thai, bem como estudos voltados para o equilíbrio na modalidade em questão. Sendo assim, sugere-se que novos estudos sejam realizados envolvendo praticantes de Muay-Thai no âmbito desta temática, no intuito de ratificar os resultados aqui apontados.

\section{CONCLUSÃO}

Conclui-se que é maior o equilíbrio quase estático na posição monopodal com olhos abertos dos praticantes de Muay Thai em nível avançado, em comparação aos de nível inicial. Este resultado demonstra a importância da continuidade do treinamento da modalidade e especialidade da mesma para ganhos de equilíbrio, que é fundamental para a prática do esporte. Esse estudo visa contribuir com os profissionais de Educação Física, principalmente interventores na modalidade do MuayThai que, com base nesses achados, poderão prescrever treinamentos sensório motores. Estes treinamentos devem objetivar o auxílio na propriocepção de indivíduos principalmente iniciantes na modalidade, primando pela melhora do equilíbrio, da evolução do seu desempenho na modalidade e consequentemente melhorando sua capacidade funcional.

\section{REFERÊNCIAS}

ALVES, P. A. M.; BARELA, J. A. Mecanismos de controle postural em crianças de 4 a 12 anos de idade e adultos. In: CONGRESSO BRASILEIRO DE BIOMECÂNICA, 11. 2005. Anais... Paraíba: [s.n.], 2005.

BANKOFF, A. D. P.; CAMPELO, T. S.; CIOL, P.; ZAMAI, C. A. Postura e equilíbrio corporal: um estudo das relações existentes. Rev Movimento e Percepção., v. 7, n. 9, 2006.

BARBATI, V. J. Teoria e prática do treinamento esportivo. 2. ed. São Paulo: Edgard Blucher, 1979.

BUCHANAN, J. A.; HORAK, B. Voluntary control of postural equilibrium patterns. Beha Brain Res., v. 143, n. 2, p. 121-140, 2003.

DUARTE, M. Análise estabilográfica da postura ereta humana quase-estática. 2000. 87 f. Tese (Livre docência 
em Biomecânica) - Universidade de São Paulo, Escola de Educação Física e Esporte, São Paulo.

DUARTE, O. A história dos esportes. 3. ed. São Paulo: Senac São Paulo, 2004.

DUARTE, M.; FREITAS, S. M. S. F. Revisão sobre posturografia baseada em plataforma de força para avaliação do equilíbrio. Rev bras fisioter., v. 14, n. 3, p. 183-192, 2010.

FALKENBACH, F. Treinamento de Muay-Thai: Bangkok x Curitiba. Rev Eletr Educ Física, 2007.

FERREIRA, F. P. M. Produção do Jornal of Biomechanics entre os anos de 2000 e 2001 relacionados ao tema equilíbrio corporal. Rio de Janeiro: Universidade do Estado do Rio de Janeiro, 2003.

IMBIRIBA, L. A.; RODRIGUES, E. C.; MAGALHÃES, J.; GARCIA, M. A. C. Informação visual e o controle do equilíbrio postural: uma abordagem de identificação de sistemas. In: CONGRESSO BRASILEIRO DE BIOMECÂNICA, 9., 2001, Gramado. Anais..., Porto Alegre: EEF-UFRGS, 2001. v. 2, p. 115-120.

KLEINER, A. F. R.; SCHLITTLER, D. X. C.; SANCHEZARIAS, M. R. O papel dos sistemas visual, vestibular, somatosensorial e auditivo para o controle postural. Rev Neuroc., v. 2, n. 19, p. 349-357, 2011.

LOPES, G. H. R. Equilíbrio unipodal e bipodal em atletas de futebol com paralisia cerebral. 2013. $74 \mathrm{f}$. Dissertação (Mestrado em Educação Física) - Universidade de Brasília, Brasília, 2013.

MANN, L.; TEIXEIRA, C. S.; LOPES, L. F. D.; MOTA, C. B. Avaliação do centro de força durante o equilíbrio estático em acadêmicos de educação física. In: MOSTRA DE INICIAÇÃO CIENTÍFICA, PÓS-GRADUAÇÃO, PESQUISA E EXTENSÃO, 5., 2005. Anais... [s.l; s.n], 2005.

MELLO, R. G. T.; GANDRA, V. D.; OLIVEIRA, L. F.; NADAL, J. Influência do Tempo de Aquisição nos Parâmetros do Sinal Estabilométrico. In: CONGRESSO BRASILEIRO DE ENGENHARIA BIOMÉDICA, 18., 2002. Anais... [s.l; s.n], 2002. v. 5, p. 406-410. para o controle postural. Rev bras fisioter, v. 19, n. 2, p. 11-18, 2006.

PAILLARD, T.; NOE, F. Effect of expertise and visual contribution on postural control in soccer. Scand J Med Sci Sports, v. 16, n. 5, p. 345-348, 2006.

ROCHA, E.; PEREIRA, R.; FLORÊNCIO, R.; TEIXEIRA, C. L. A.; PENHA, J. C. L.; NASCIMENTO, M.; MADUREIRA, F. Análise cinemática do chute circular no muaythai em praticantes iniciantes da modalidade. Lecturas, Educación Física y Deportes, Revista Digital, v. 18, n. 183, 2013.

ROUGIER, P. Visual feedback induces opposite effects on elementary centre of gravity and centre of pressure minus centre of gravity motions in undisturbed upright stance. Clin Biomech (Bristol, Avon), v. 18, n. 3, p. 341349, 2003.

TEIXEIRA, C. S.; LOPES, L. F. D.; MOTA, C. B.; ROSSI, A. G. Utilização da visão para a manutenção do equilíbrio estático em jovens. The FIEP Bulletin, v. 77, p. 636-639, 2007.

VIEIRA, T. M. M.; OLIVEIRA, L. F. Equilíbrio postural de atleta remadores. Rev Bras Med Esporte, v. 12, n. 3, p. 135-138, 2006.

YOSHITOMI, S. K.; TANAKA, C.; DUARTE, M.; LIMA, F.; MORYA, E.; HAZIME, F. Respostas posturais à perturbação externa inesperada em judocas de diferentes níveis de habilidade. Rev Bras Med Esporte, v. 12, n. 3, p. 159163, 2006.

Recebido em: 13 de abril de 2016 Versão final recebida em: 03 de maio de 2016 Aceito em: 15 de maio de 2016

MOCHIZUKI, L.; AMADIO, A. C. As informações sensoriais 